\title{
Ion acceleration and D-D fusion neutron generation in relativistically transparent deuterated nanowire arrays
}

\author{
A. Curtis, ${ }^{1}$ R. Hollinger $\odot,{ }^{1,}{ }^{*}$ C. Calvi, ${ }^{2}$ S. Wang, ${ }^{1}$ S. Huanyu $\odot,{ }^{1}$ Y. Wang, ${ }^{1}$ A. Pukhov, ${ }^{3}$ V. Kaymak, ${ }^{3}$ \\ C. Baumann ${ }^{\circ},{ }^{3}$ J. Tinsley ${ }^{10},{ }^{4}$ V. N. Shlyaptsev, ${ }^{1}$ and J. J. Rocca ${ }^{1,2, \dagger}$ \\ ${ }^{1}$ Electrical and Computer Engineering Department, Colorado State University, Fort Collins, Colorado 80523, USA \\ ${ }^{2}$ Physics Department, Colorado State University, Fort Collins, Colorado 80523, USA \\ ${ }^{3}$ Institut für Theoretische Physik I, Heinrich-Heine-Universität Düsseldorf, 40225 Düsseldorf, Germany \\ ${ }^{4}$ Mission Support \& Test Services, LLC (MSTS)
}

(Received 31 December 2020; accepted 6 October 2021; published 15 December 2021)

\begin{abstract}
Laser irradiation of deuterated polyethylene nanowire arrays at intensities of $5 \times 10^{19} \mathrm{~W} \mathrm{~cm}^{-2}$ was recently reported to accelerate deuterons to multi-MeV energies, resulting in microscale fusion. Here we show that irradiation of deuterated nanowire targets at intensities of $\sim 3 \times 10^{21} \mathrm{~W} \mathrm{~cm}^{-2}$ with high contrast $\lambda=400 \mathrm{~nm}$ laser pulses of 45 fs duration leads to different plasma dynamics in which the tip of the nanowires rapidly explodes to form an overdense plasma, but the onset of relativistic transparency allows the ultrashort laser pulse to penetrate deep into the nanowire array, enhancing particle acceleration. Experiments and particle-in-cell simulations show that ions are accelerated in the laser beam backward direction to energies up to $13 \mathrm{MeV}$ by a target normal sheath acceleration (TNSA) field that develops in the front of the target, forming collimated beams characterized by full wave at half maximum divergence as low as $7.5^{\circ}$. The simulations also show that deeper within the nanowire array ions are accelerated radially to $\mathrm{MeV}$ energies by an internal TNSA field normal to the nanowire surfaces. These radially accelerated deuterons collide with other $\mathrm{D}$ atoms, as do those directed toward the $\mathrm{CD}_{2}$ substrate, leading to D-D fusion reactions. Irradiation with $8 \mathrm{~J}$ laser pulses is measured to generate up to $1.2 \times 10^{7} \mathrm{D}$-D fusion neutrons per shot. The simulations suggest the use of a thinner substrate would also accelerate ions originating from a small area in the back of the target in the laser forward direction.
\end{abstract}

DOI: 10.1103/PhysRevResearch.3.043181

\section{INTRODUCTION}

Progress in high energy, directional ion production from laser produced plasmas has closely followed the development of high intensity chirped pulse amplification laser systems. A few early experiments reached $\mathrm{MeV}$ ion energies per nucleon in the 1980s and 1990s [1,2] using bulk and thin targets. Three simultaneous experiments in the year 2000 [3-5] reported on the so-called target normal sheath acceleration (TNSA) mechanism [6,7] demonstrating several tens of $\mathrm{MeV}$ ions and various degrees of collimation with decreased divergence as a function of increasing ion energy, leading to a broad expansion of interest in the field. TNSA is a process in which ions are accelerated by a large electrostatic field $(\mathrm{GV} / \mathrm{mm})$ due to space charge buildup caused by the displacement of electrons accelerated to relativistic velocities by the $\mathbf{j} \times \mathbf{B}$ force in the laser field. In traditional forward acceleration TNSA the accelerated electrons traverse the target creating

\footnotetext{
*reed.hollinger@gmail.com

†jorge.rocca@colostate.edu
}

Published by the American Physical Society under the terms of the Creative Commons Attribution 4.0 International license. Further distribution of this work must maintain attribution to the author(s) and the published article's title, journal citation, and DOI. an electrostatic "sheath" field as they propagate away from the target, ionizing the target rear surface and accelerating the resulting ions in the laser forward direction. As a consequence of this, the primary condition for traditional TNSA is a laser intensity sufficient to drive the electrons through the remaining solid target [8]. More recent experiments with higher intensity, energetic lasers resulted in proton energies up to 85 [9] or $94 \mathrm{MeV}$ [10] from pulses with duration exceeding $0.5 \mathrm{ps}$ and intensity of $(0.7-3) \times 10^{20} \mathrm{~W} \mathrm{~cm}^{-2}$ on the target.

A variety of other acceleration mechanisms have been explored demonstrating $\mathrm{MeV}$ energy ion beams emanating from the rear side of foil targets and propagating in the laser beam forward direction. Among these mechanisms is radiation pressure acceleration (RPA) [11], where at sufficiently high laser intensity strong radiation pressure (or ponderomotive effect) drives plasma electrons into the target, creating the electrostatic field conditions for ion acceleration. Two demonstrated subcategories of RPA are referred to as "light sail" in thin targets [12,13], and "hole boring acceleration" in thick targets [14]. A process that occurs in some of the plasmas of interest for ion acceleration is relativistically induced transparency (RIT) [15]. This phenomenon occurs when an overdense plasma becomes transparent to the laser pulse due to the increase in the mass of relativistic electrons, which decreases the plasma frequency and therefore the effective critical electron density $[16,17]$. In the case of ultrathin foil targets, the expansion of the foil may lead to the onset of RIT, 
which can result in enhanced ion acceleration, a regime known as "breakout afterburner acceleration" $[18,19]$.

Front side acceleration of ions into the laser backward direction is of particular interest to the present work. Published results for different target configurations producing front side deuteron acceleration include deuterium clusters [20], heavy water droplets [21], frozen heavy water solid targets [22], foils [23,24], and microstructured targets [25]. The most cited acceleration mechanism is $\mathbf{j} \times \mathbf{B}$ heating of the charged particles in the laser field. An experiment in which thin Mylar foils were irradiated at an intensity of $5 \times 10^{18} \mathrm{~W} \mathrm{~cm}^{-2}$ with a $>10^{10}$ contrast pulse [26] produced 4.5 $\mathrm{MeV}$ protons. The highest proton energy reported from front side acceleration is $18 \mathrm{MeV}$ [27]. Some of these experiments also investigated the generation of D-D fusion neutrons [20-22,25,28]. D-D fusion neutrons have also been recently produced by irradiating arrays of aligned nanowires [29]. Similar to nanoclusters, nanowire arrays have the advantage of efficient volumetric heating but offer the possibility of creating a medium with several orders of magnitude higher average plasma density. Femtosecond laser pulses can penetrate deep into the nanowire array, depositing nearly their full energy before a critical layer is formed at the top of the nanowire array. Once the laser energy is deposited, the heated wires explode, and ions are accelerated to $\mathrm{MeV}$ energies while the gap between the wires is filled with a semihomogeneous near solid density plasma with multi-keV temperatures [30-32]. Efficient absorption [33] of femtosecond laser pulses of relativistic intensity into ordered nanowire arrays leads to extreme degrees of ionization [30,31], enhanced conversion efficiency of optical laser light into $x$ rays [30,34-36], and the acceleration of electrons to tens of MeVs [37,38]. We have recently irradiated arrays of high aspect ratio deuterated polyethylene $\left(\mathrm{CD}_{2}\right)$ nanowires with high contrast pulses of $1.6 \mathrm{~J}$ energy and $60 \mathrm{fs}$ duration focused to an intensity of $5 \times 10^{19} \mathrm{~W} \mathrm{~cm}^{-2}$, accelerating deuterons to $3 \mathrm{MeV}$, generating up to $2 \times 10^{6}$ D-D fusion neutrons per shot [29].

Here we report experiments observing laser backward acceleration of ions originating from aligned deuterated nanowire arrays with nearly two orders of magnitude higher laser intensities, up to $3 \times 10^{21} \mathrm{~W} \mathrm{~cm}^{-2}$. Fully relativistic three-dimensional particle-in-cell (PIC) simulations show that despite the tip of the wires exploding early in the laser pulse and forming an overdense plasma, RIT allows the laser light to still penetrate deep into the array. This in turn extends the region of interaction, which generates the relativistic electron currents required to create the large TNSA field that drives the front side acceleration. Experiments show ions are accelerated in the laser backward direction to energies up to $13 \mathrm{MeV}$, forming well collimated beams characterized by full wave at half maximum (FWHM) divergence as low as $7.5^{\circ}$. Laser pulses of $8 \mathrm{~J}$ are measured to generate up to $1.2 \times 10^{7} \mathrm{D}$-D fusion neutrons per shot.

\section{EXPERIMENTS AND RESULTS}

The experiments were performed using Colorado State University's ALEPH petawatt-class titanium sapphire laser [39]. Deuterated polyethylene nanowire arrays were irradiated at relativistic intensities up to $3 \times 10^{21} \mathrm{~W} \mathrm{~cm}^{-2}$ with
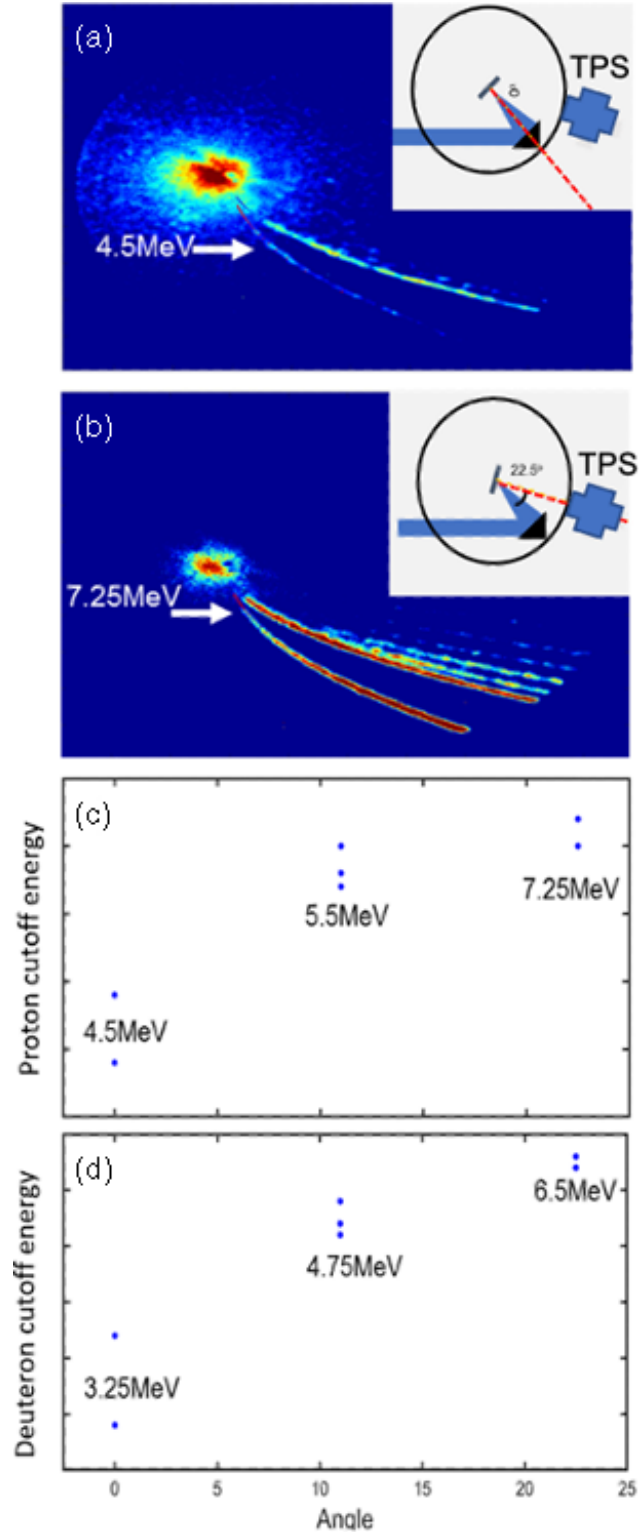

FIG. 1. Thomson parabola traces for ions at two different angles with respect to the target normal. (a) TPS at $22.5^{\circ}$ off-target normal allowing for normal impingement of the laser pulse onto the target. (b) TPS traces normal to the target and laser pulse impinging at $22.5^{\circ}$ off target normal. (c) Maximum proton energy as a function of irradiation angle. (d) Maximum deuteron energy as a function of irradiation angle.

frequency doubled $\lambda=400 \mathrm{~nm}$ laser pulses. Pulses of up to $8 \mathrm{~J}$ of energy and $45 \mathrm{fs}$ in duration were focused into a $\sim 1.6 \mu \mathrm{m}$ FWHM diameter spot with an $f / 2$ off-axis parabola. Frequency doubling in a thin potassium dihydrogen phosphate (KDP) crystal followed by five dichroic mirrors with $>99.5 \%$ transmission at $800 \mathrm{~nm}$ yielded a contrast of $>10^{12}$, which prevents the creation of a preplasma and damage to the nanowires prior to the arrival of the high intensity pulse, even in the case of the highest intensities used in the experiments. The nanowire array targets were grown by heated extrusion into nanoporous alumina templates [40]. The targets used in the experiment were arrays of $200 \mathrm{~nm}$ diameter $\mathrm{CD}_{2}$ 

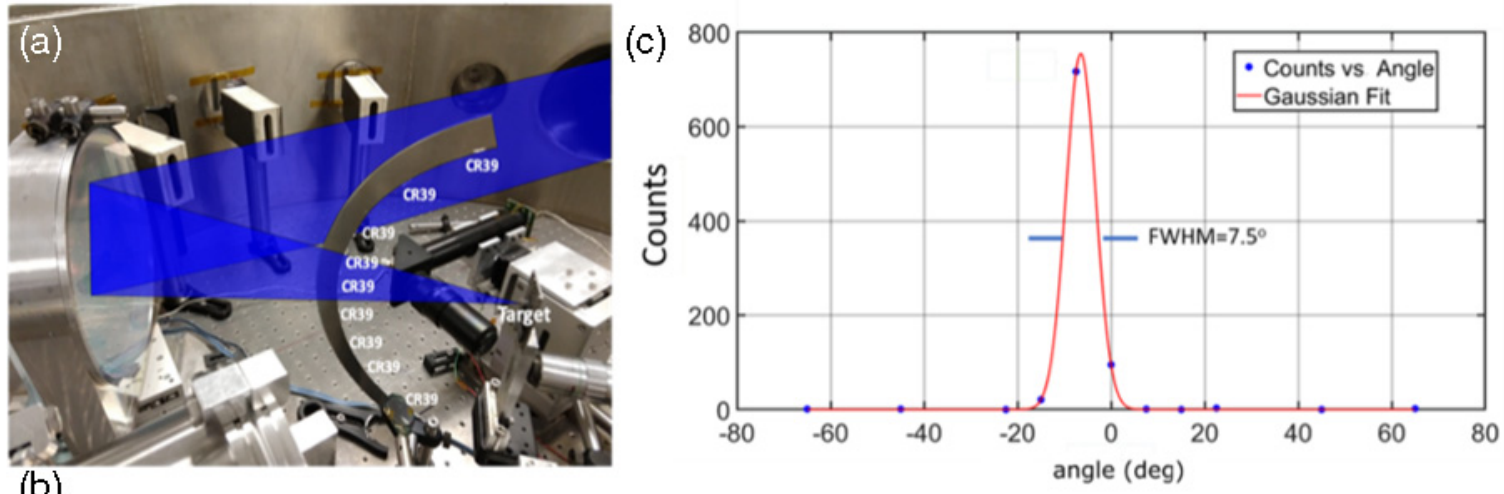

(b)
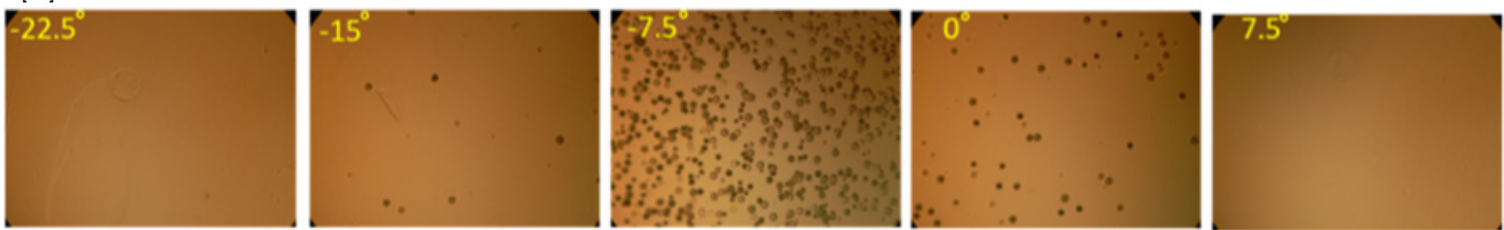

FIG. 2. (a) Setup to measure energy and angular distribution of ions accelerated in the laser backward direction using an array of filtered CR-39 detectors. (b) Microscope images of ion traces in developed CR-39. The data were obtained with the $250 \mu \mathrm{m} \mathrm{Cu}$ filter. The small displacement in the maximum ion flux from the laser propagation axis is due to target tilt in the vertical axis. (c) Angular distribution of ions with energy $>13 \mathrm{MeV}$. The ions are confined to a $7.5^{\circ} \mathrm{FWHM}$ emission cone.

nanowires, 5-10 $\mu \mathrm{m}$ in length with an average density of $19 \%$ solid density corresponding to an average wire to wire distance of $\sim 400 \mathrm{~nm}$, supported by a $200-300 \mu \mathrm{m}$ thick $\mathrm{CD}_{2}$ substrate. The data presented here is for arrays of $5 \mu \mathrm{m}$ long nanowires, unless indicated. Targets were mounted in a vacuum chamber where high energy ion diagnostics included a Thomson parabola spectrometer (TPS) with a microchannel plate (MCP)/phosphor screen detector, and filtered CR-39 track detectors. The neutrons were detected with an array of four time-of-flight (TOF) scintillator-photomultiplier neutron

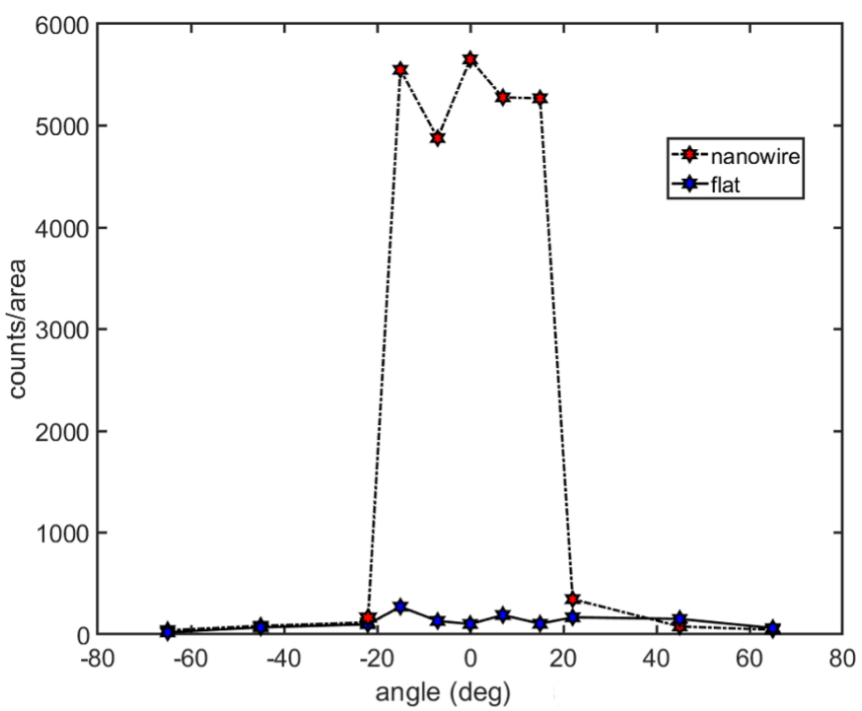

FIG. 3. CR-39 ion flux angular distribution from an array of $\mathrm{CD}_{2}$ nanowires (red stars) compared to that from a flat $\mathrm{CD}_{2}$ target (blue stars) demonstrating the increased ion flux and directionality characteristics of the nanowires. The measurement was conducted using $100 \mu \mathrm{m} \mathrm{Cu}$ filters, which select ion energies $>7.5 \mathrm{MeV}$. detectors. The sensitivity of the detectors was calibrated using the known neutron flux from a dense plasma focus machine.

To measure ion energy spectra the TPS was placed $1.2 \mathrm{~m}$ from the plasma at an angle of $22.5^{\circ}$ from the laser axis with the laser impinging at either normal incidence [Fig. 1(a)], $11.25^{\circ}$, or $22.5^{\circ}$ with respect to the target normal, such that in the latter case the target normal is aligned with the axis of the TPS [Fig. 1(b)]. The energy along the $\mathrm{D}$ and $\mathrm{H}$ ion traces in the TPS was calibrated using an ion TOF method in which the MCP is gated for different time delays [29] and was confirmed with SIMION simulations [41]. The cutoff ion energy in the TPS traces was found to increase as the target normal was rotated toward the TPS. A proton energy exceeding $7 \mathrm{MeV}$

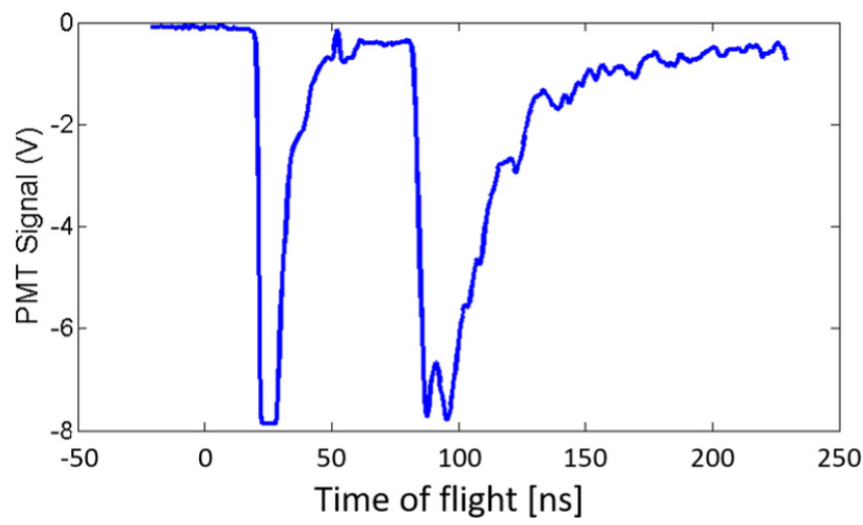

FIG. 4. Oscilloscope trace from a scintillator/PMT neutron TOF detector on a deuterated nanowire array target $10 \mu \mathrm{m}$ in length irradiated with an intensity of $3 \times 10^{21} \mathrm{~W} / \mathrm{cm}^{2}$. The peak at the left is from the plasma $x$ ray/gamma ray burst and the second broader peak is the neutron signal. The integrated D-D neutron yield from four TOF detectors for this shot is $1.2 \times 10^{7}$ neutrons 


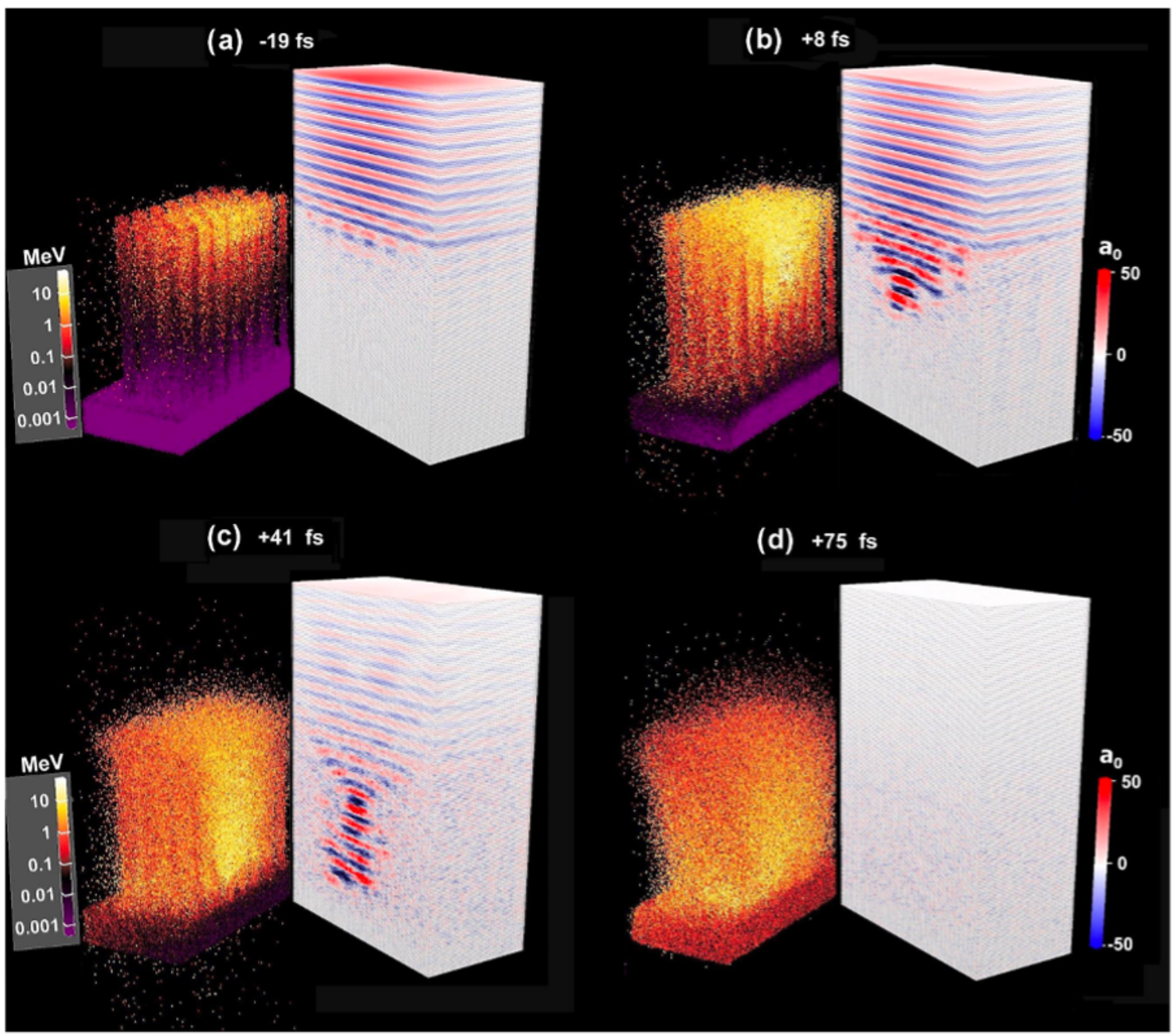

FIG. 5. Three-dimensional PIC simulation of the evolution of the density and energy of free electrons (left) and the incident laser electric field (right) into the $\mathrm{CD}_{2}$ nanowire array irradiated with a $45 \mathrm{fs}$ FWHM duration laser pulse at an intensity of $4 \times 10^{21} \mathrm{~W} \mathrm{~cm}^{-2}$. Frame times are with respect to arrival of the peak of the laser pulse at the tip of the nanowire array. In $(b, c)$ the laser pulse is seen to penetrate into the nanowire array due to relativistic transparency, which extends the interaction length.

was detected in the target normal direction (target rotated $22.5^{\circ}$ with respect to the laser beam).

Of particular interest is the ion distribution at shallow angles with respect to target normal when the laser beam axis is aligned with the nanowires. However, the TPS cannot be used to diagnose the ion energy when the target is irradiated at normal incidence, as the line of sight is blocked by the off-axis parabola. The ion beam peak energy and directionality for normal incidence irradiation was instead measured using an array of filtered CR-39 detectors mounted on a thin curved stainless steel "sickle" positioned directly in the laser beam path at $10 \mathrm{~cm}$ from the target, as shown in Fig. 2(a). The mount was $1 \mathrm{~mm}$ thick to avoid blocking a significant fraction of the incident laser beam. Eleven CR-39 detectors were positioned on it at different angles ranging from $-65^{\circ}$ to $+65^{\circ}$ with respect to the beam axis as shown in Fig. 2(a). Copper filters of 100,250 , and $300 \mu \mathrm{m}$ thickness with deuteron cutoff energies of 8,13 , and $15 \mathrm{MeV}$, respectively, were mounted on top of the CR-39. No ions were detected using the $300 \mu \mathrm{m}$ thick filters. The maximum ion energy of $13 \mathrm{MeV}$ was observed using the $250 \mu \mathrm{m}$ filter. The maximum flux was observed near the target normal [Figs. 2(b) and 2(c)]. Only small ion track signals were observed at the surrounding angles. These data indicate that the ions with the greatest energy are highly directional, contained within a $7.5^{\circ} \mathrm{FWHM}$ cone. A comparison between the nanowire target and a solid density $\mathrm{CD}_{2}$ flat target irradiated with the same laser pulses is shown in Fig. 3. To be able to detect the lower ion flux associated to the solid flat target's thinner $100 \mu \mathrm{m} \mathrm{Cu}$ filters were used, which saturated the central CR-39 track detectors for the ion flux produced by the nanowires. What is clear from this figure is the large increase of flux and directionality of the ions at this energy from the nanowire targets in comparison with the flat solid target.

The D-D fusion neutron flux generated was measured using four time-of-flight scintillator-photomultiplier detectors placed at different angles and distances from the target normal. Figure 4 shows the x-ray and neutron signals from a single detector. The time-of-flight separation for the $\mathrm{x}$-ray pulse and neutron peaks consistently indicated that the energy of the neutrons from all four detectors placed at different distances from the target is the $2.45 \mathrm{MeV}$ characteristic of 


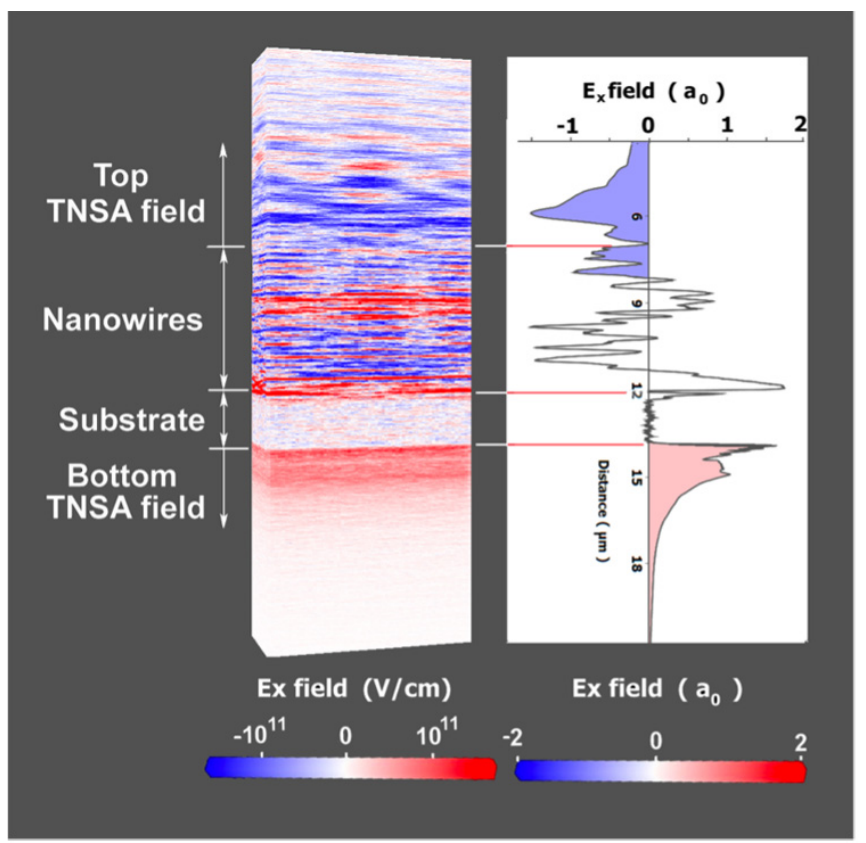

FIG. 6. Electric field distribution in the nanowire axial (longitudinal) direction (in linear scale) in an array of $200 \mathrm{~nm}$ diameter, 19\% solid density $\mathrm{CD}_{2}$ nanowires at $30 \mathrm{fs}$ after arrival of the peak of the 45 fs FWHM laser pulse to the tip of the nanowires. A TNSA field develops near the top of the array that accelerates ions in the laser backward direction.

D-D fusion reactions. By integrating the neutron signals from all four detectors the generation of up to $1.2 \times 10^{7}$ neutrons per shot into $4 \pi$ sr was measured. Previous work conducted at pulse energies up to $1.65 \mathrm{~J}$ and intensities up to $2 \times$ $10^{20} \mathrm{~W} \mathrm{~cm}^{-2}$ found a rapid supralinear increase in the number of neutrons generated as a function of laser pulse energy [29]. Our results covering a range of intensities above this value and up to $3 \times 10^{21} \mathrm{~W} \mathrm{~cm}^{-2}$ find that the increase in the number of neutrons in this higher intensity range is approximate linear.

\section{SIMULATIONS AND DISCUSSION}

Fully relativistic three dimensional particle-in-cell (PIC) simulations were performed using the VIRTUAL LASER PLASMA LABORATORY code [42] to gain understanding of the ion acceleration mechanism. The code includes relevant physics packages for optical field ionization, binary collisions, and sequential electron impact ionization. The simulations considered an array of $\mathrm{CD}_{2}$ nanowires $200 \mathrm{~nm}$ in diameter and $5 \mu \mathrm{m}$ in length at $19 \%$ of solid density, irradiated by a high contrast $\lambda=400 \mathrm{~nm}$ laser pulse of $45 \mathrm{fs}$ in duration with an intensity of $4 \times 10^{21} \mathrm{~W} \mathrm{~cm}^{-2}$ impinged parallel to the wires. They were conducted with initially three particles per cell. The number of electrons grows to as many as eight per cell when the atoms are ionized. The cell size was $0.05-0.1$ wavelengths. The nanowire array was simulated by either modeling a single nanowire with periodic boundaries in the transverse plane creating a mirror symmetry to synthesize the array, or an array of multiple wires. The multiple wire simulations used more realistic nanowire target settings with up to 170 individual nanowires within the relevant irradiated region.

The simulations show that the dynamics differs from that studied in $\mathrm{CD}_{2}$ nanowire arrays at much lower irradiation intensity [29]. Figure 5 shows maps of the free electron density, electron energy, and the laser electric field at four different times with respect to the arrival of the peak of the laser pulse to the tip of the nanowires. At the highly relativistic intensities of the present study the tips of the nanowires

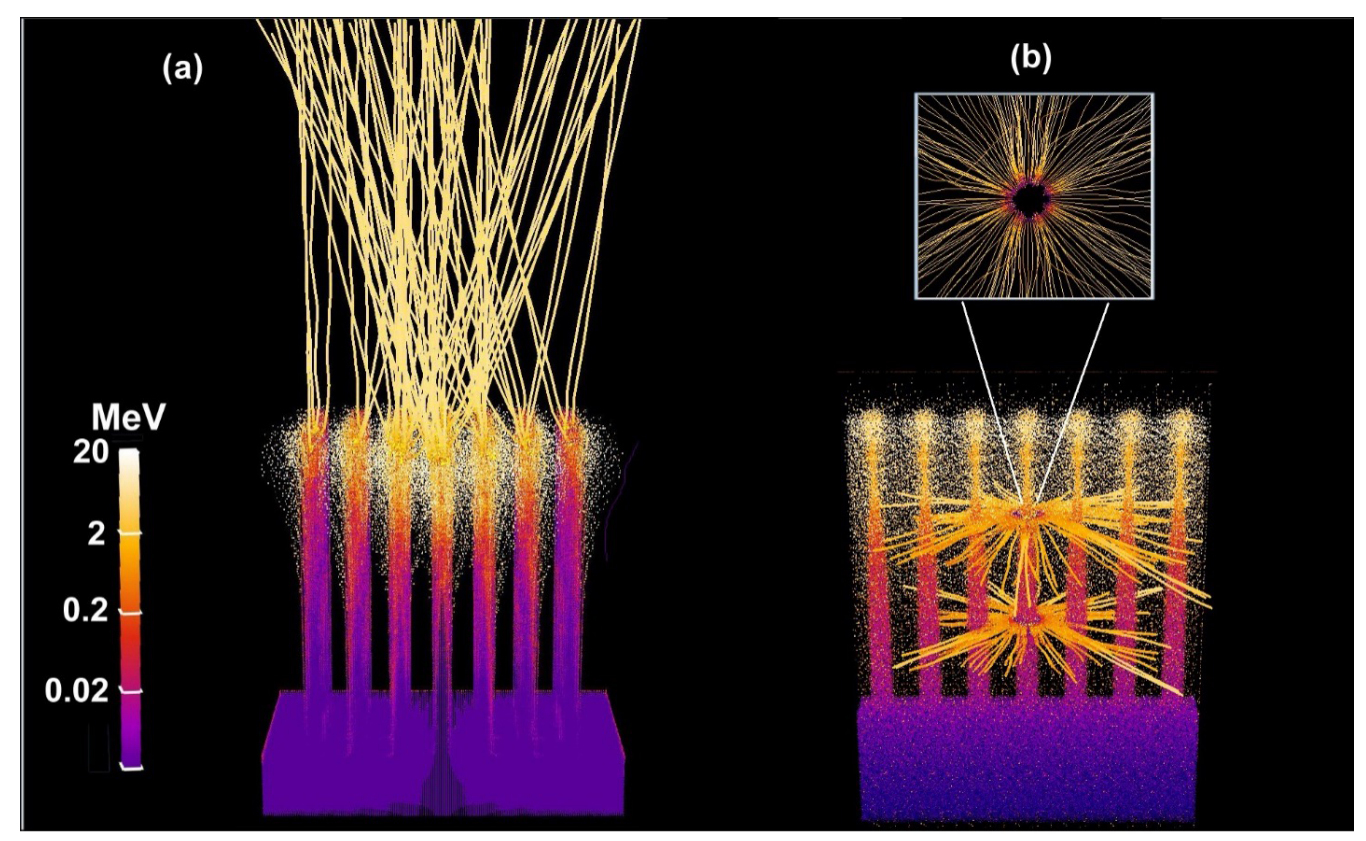

FIG. 7. PIC simulations of ion acceleration in an array of $200 \mathrm{~nm}$ diameter $\mathrm{CD}_{2}$ nanowire irradiated at an intensity of $4 \times 10^{21} \mathrm{~W} \mathrm{~cm}{ }^{-2}$ with a 45 fs laser pulse. (a) Trajectories of deuterons from the exploding nanowire tips accelerated in the laser backward direction; (b) trajectories of radially accelerated ions which lead to D-D fusions. 
rapidly explode as illustrated by the electron energy maps in Fig. 5(a), corresponding to a time of $19 \mathrm{fs}$ prior to the arrival of the peak of the laser pulse to the tip of the nanowires. The plasma in the interwire gaps becomes overdense, precluding further penetration of the laser pulse deep into the array. However, as the laser intensity increases toward its peak value, relativistic transparency takes place [Fig. 5(b)]. At an intensity of $4 \times 10^{21} \mathrm{~W} \mathrm{~cm}^{-2}$ relativistic effects increase the critical electron density by a factor $\gamma \sim 15$ to $n_{\text {ecr }} \sim 10^{23} \mathrm{~cm}^{-3}$ $\left(n_{\mathrm{ecr}}=\gamma n_{\mathrm{ec}}\right.$, where the relativistic factor is $\gamma=\sqrt{1+\frac{a_{o}^{2}}{2}}$ and the normalized vector potential is $a_{o}=21.5$ ). This increase in the critical density makes it possible for the laser pulse to penetrate deep into the overdense $\mathrm{CD}_{2}$ nanowire array plasma [Fig. 5(c) at a time of $+41 \mathrm{fs}$ )], approaching, but not surpassing the critical density inside the substrate. Electrons are extracted from the nanowires by optical field ionization mostly perpendicular to the wire axis. These electrons are accelerated by the laser field toward the target substrate, creating a displacement of charge that leaves the nanowire tips highly positively charged. Subsequently, a strong quasistatic electric field accelerates the electrons from the substrate in the laser backward direction, creating an electron return current which propagates along the entire length of the nanowires to balance the charge separation. During this process which lasts 50-100 fs the charge separation creates an electric field in front of the nanowire array that accelerates the ions from the tips in the backward direction toward the laser (Fig. 6). The strength of this axial target normal sheath acceleration (TNSA) field on front of the target reaches $2 \times 10^{11} \mathrm{~V} / \mathrm{cm}$. This field is an order of magnitude smaller than the laser field. However, because it is a quasistatic field and has a spatial dimension larger than the dephasing length within the nanostructure plasma, it dominates in accelerating the deuterons to large energies. The PIC simulations agree with the experiment in depicting the formation of a well collimated ion beam in the laser backward direction, in which the most energetic ions have a low divergence of $<10^{\circ}$ FWHM, [Fig. 7(a)]. The simulated electron energy spectra, and the corresponding spectra of ions accelerated in the laser backward direction are shown in Fig. 8 for three different times with respect to the arrival of the peak of the laser. The hot electron temperature near the time of arrival of the peak of the laser pulse is computed to reach $3.7 \mathrm{MeV}$. The ion cutoff energy is computed to reach $\sim 24 \mathrm{MeV} 140$ fs later, which is in reasonable agreement with the experiment, taking into account the limited dynamic range of the measurement. Using these values in the TNSA relationship between ion cutoff energy and hot electron temperature $E_{\text {ion }}=\alpha T_{\text {hot }}$ used in the literature [6,7], we find a value of $\alpha \sim 6.5$. In the range of laser parameters investigated (normalized vector potential $a_{o}=5-20$ ), the ion cutoff energy is also found to be approximately proportional to the $a_{o}$, as in regular TNSA ponderomotive scaling.

Besides this front TNSA field external to the nanowires which accelerates ions backward toward the laser, the simulations show there also exists an internal TNSA field in the nanowire array that accelerates the ions radially. This internal field is also a quasistatic field but in the radial nanowire direction. This radial field that surrounds each of the nanowires is formed due to the displacement of electrons from the
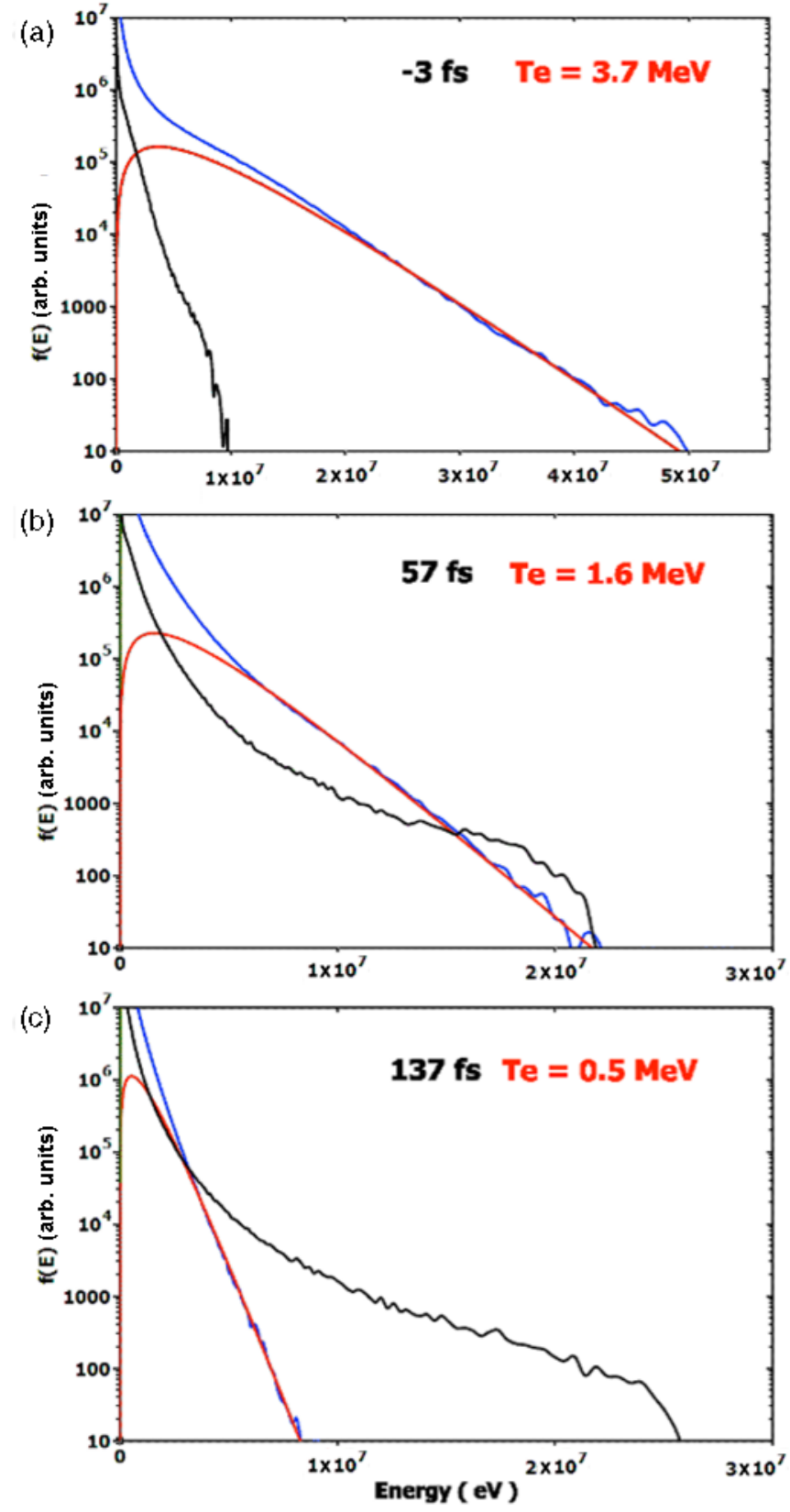

FIG. 8. Simulated electron (blue) and proton (black) spectra computed from PIC simulations at three different times with respect to the peak of the laser pulse: (a) $-3 \mathrm{fs},(\mathrm{b})+57 \mathrm{fs}$, and (c) $+137 \mathrm{fs}$. A fit of the Maxwell Boltzmann distribution for the electrons is plotted in red with the temperature in the inset.

nanowire surfaces into the voids by the laser electric field (so-called Brunel electrons). Because the effective acceleration length from the displacement of the positively charged nanowires and negatively charged voids is half of the interwire separation, the ions are accelerated to only $\sim 10 \%-20 \%$ percent of the energy of ions at the tip. The radial acceleration due to the internal TNSA field is visible in Fig. 7(b). Ions only gain energy in the vicinity close to the nanowire surface after which they move in the radial direction scattering and dissipating their energy. Because the external TNSA field acceleration is much larger than the radial acceleration ions at the tip have 

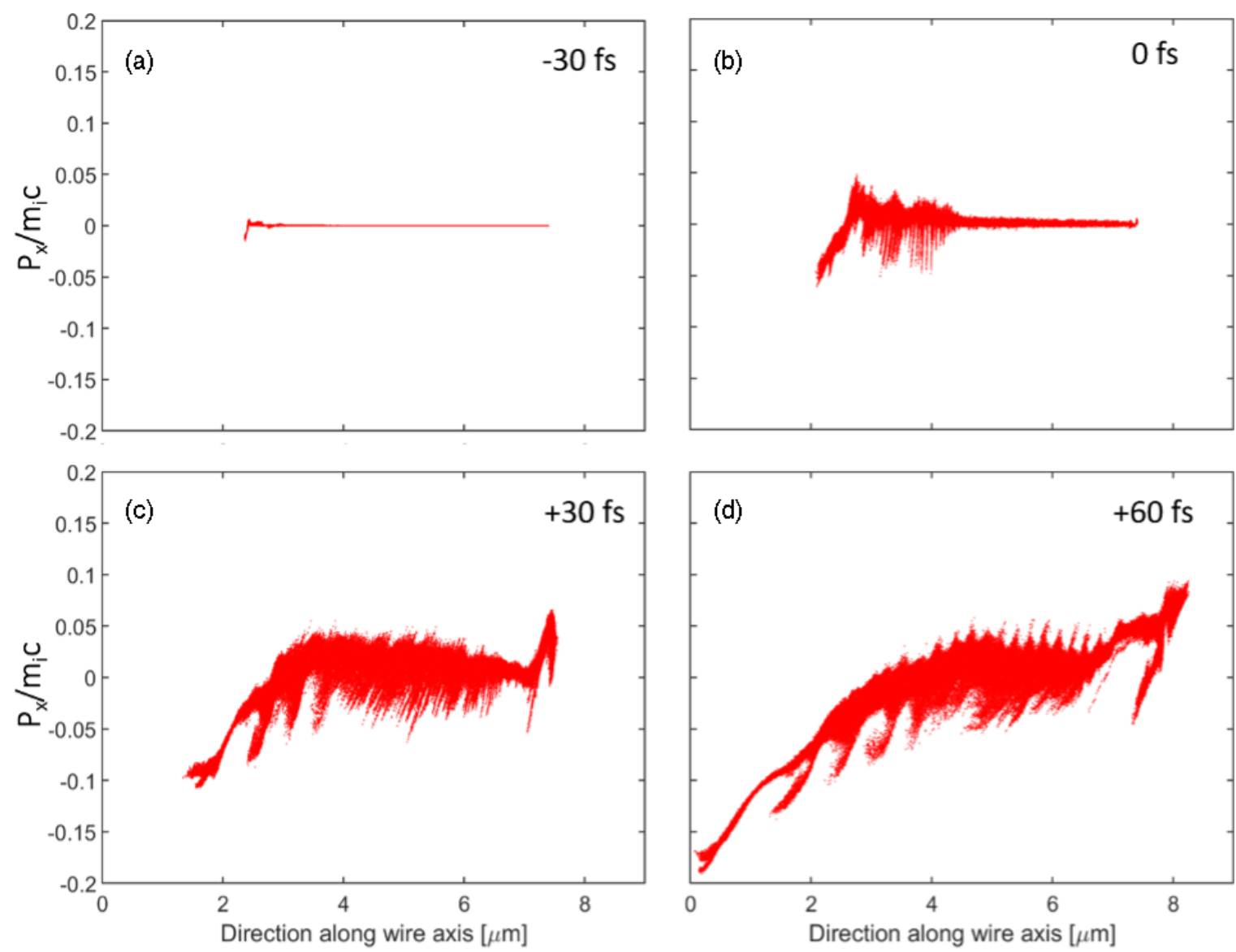

FIG. 9. One-dimensional phase space plots of ion momenta at different times with respect to the time of arrival of the peak of the laser pulse to the tip of the nanowires. Negative values represent momenta in the laser backward direction. The modulations in (c), (d) contain only a small fraction of the ions. The elongated feature on the bottom left of (d) contains ions originating from the nanowire tips, accelerated by a quasistatic field created by the laser. The rest are created by the laser field and the quasistatic field, with a periodicity related to the laser wavelength divided by the index of refraction of the plasma.

a narrow angle of divergence. Deep into the nanowire array, an internal axial field visible as a red region adjacent to the target in Fig. 6 bends the radially accelerated deuterons toward the $\mathrm{CD}_{2}$ substrate. These deuterons collide with the substrate, adding to the generation of neutrons. The number of deuterons accelerated to $>0.2 \mathrm{MeV}$ at angles between $0^{\circ}$ (target normal in the forward direction) and $90^{\circ}$ contributing to D-D fusion is computed to be $3.8 \times 10^{12}$, while the number accelerated to $>1 \mathrm{MeV}$ is computed to be $3 \times 10^{11}$. The corresponding computed deuteron phase space is shown in Fig. 9 for four different times: (a) 30 fs preceding the arrival of the peak of the laser pulse to the tip of the array, (b) at the time of arrival of the peak of the laser pulse, and (c,d) 30 and 60 fs following the peak of the laser pulse. The ions are drawn out of the tip of the wire and gain momentum due to the creation of the front TNSA field. The negative momenta represent motion toward the direction of the incoming laser.

A simulation conducted for laser pulse energies up to 8 $\mathrm{J}$ impinging on an array of $10 \mu \mathrm{m}$ long nanowires estimated the generation of $\sim 2 \times 10^{7}$ neutrons, in reasonable agreement with the experimental measurement of more than half that value. The deuteron momentum distribution was computed with the PIC code. The number of neutrons generated per deuteron was subsequently computed based on the stopping power of deuterons in the $\mathrm{CD}_{2}$ nanowires and in the substrate. The contribution to neutron production from deuterons colliding with the 200-300 $\mu \mathrm{m}$ thick substrate layer that supports the nanowires is important. However, the dependence on target thickness is less so because the majority of the deuterons impinge at large angles with respect to the surface normal and have energies below $1 \mathrm{MeV}$ which are stopped within less than 10-20 microns in the $\mathrm{CD}_{2}$ substrate. The simulation, shown in Fig. 10, indicates that initially the number of neutrons produced increases supralinearly as a function of laser pulse energy, as previously reported in Ref. [28] for laser pulse energies up to $3.5 \mathrm{~J}$, corresponding to an intensity of up to $1.6 \times 10^{20} \mathrm{~W} \mathrm{~cm}^{-2}$. The supralinear growth of neutron creation at the lower intensities is associated with the fast growth of the D-D reaction cross section with increased D ion energy. However, in the higher intensity range investigated here the growth of the number of neutrons with laser energy is computed to progressively decrease into linear, in agreement with the experiments. At energies above $5 \mathrm{~J}$, the dependence becomes practically linear as shown by the slopes in a spline fit to the numerical data.

The simulations also predict that in the case in which a thinner substrate was to be used, a high energy ion beam propagating in the laser forward direction would also be gen- 


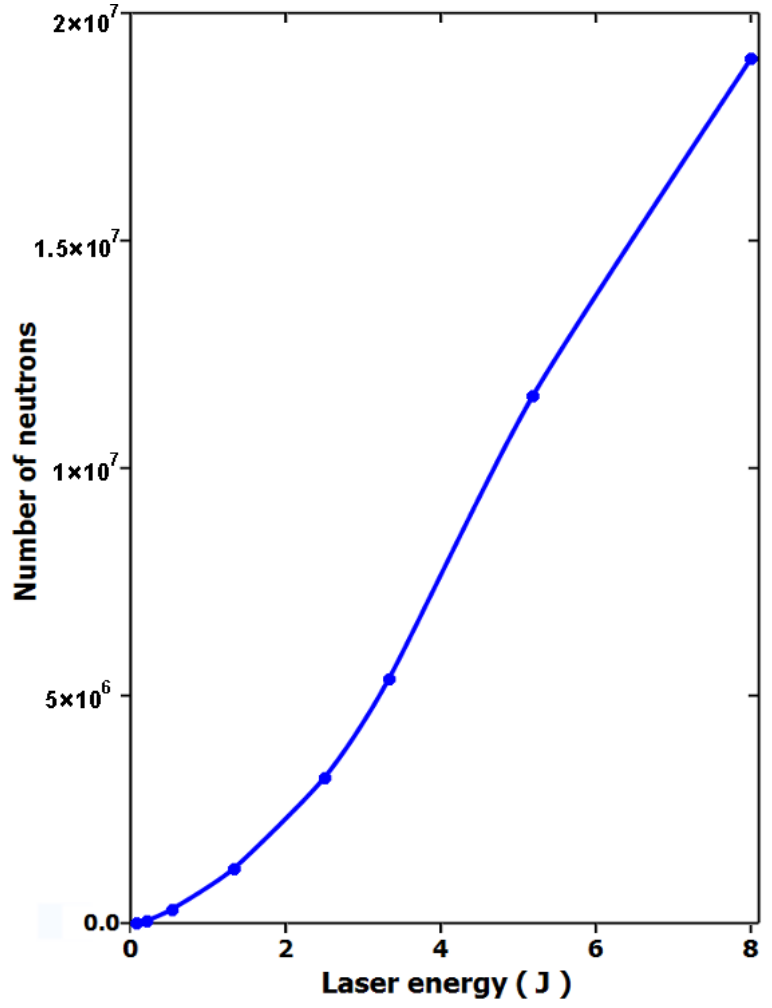

FIG. 10. Computed neutron number as a function of laser pulse energy. The increase in neutron production changes from supralinear to increasingly linear.

erated from a TNSA field generated on the back surface of the target. Figure 11 shows the results of a simulation for the nanostructure parameters and laser irradiation intensity investigated in this work, but assuming a $\mathrm{CD}_{2}$ target substrate thickness of $1 \mu \mathrm{m}$. In practice, the thin plastic substrates would be supported by an electroformed metal mesh. The electrons accelerated by the laser field in the forward direction traverse the thin substrate generating a quasistatic TNSA field on the back of the target, where ions are accelerated in the forward direction. While the magnitude of this bottom TNSA electric field is several times smaller than the laser and surface wave fields that create charge displacement and radial acceleration, it has an extent of the order of several microns, much larger than the submicron interwire distance, hence accelerating ions to higher energy. Energies up to $20 \mathrm{MeV}$ are predicted for protons, which could be further increased, optimizing the nanowire parameters. For the specific laser and nanowire parameters of this work, this forward propagating ion beam is computed to emanate from a very small area (submicron diameter) at the bottom of the nanowires and at the rear of the substrate, which allows the generation of highly collimated or tightly focused deuteron/proton beams of specific energy with the use of a magnetic lens. This small source size is a consequence of relativistic transparency, which enhances the penetration of the most intense central part of the laser beam relative to the wings. This additional effect occurs because since the index of refraction, $n=1-\sqrt{\frac{n_{e}}{\gamma n_{c}}}$, has its maximum on the axis, refraction deflects the light toward the center. This process of strong self-focusing sig-

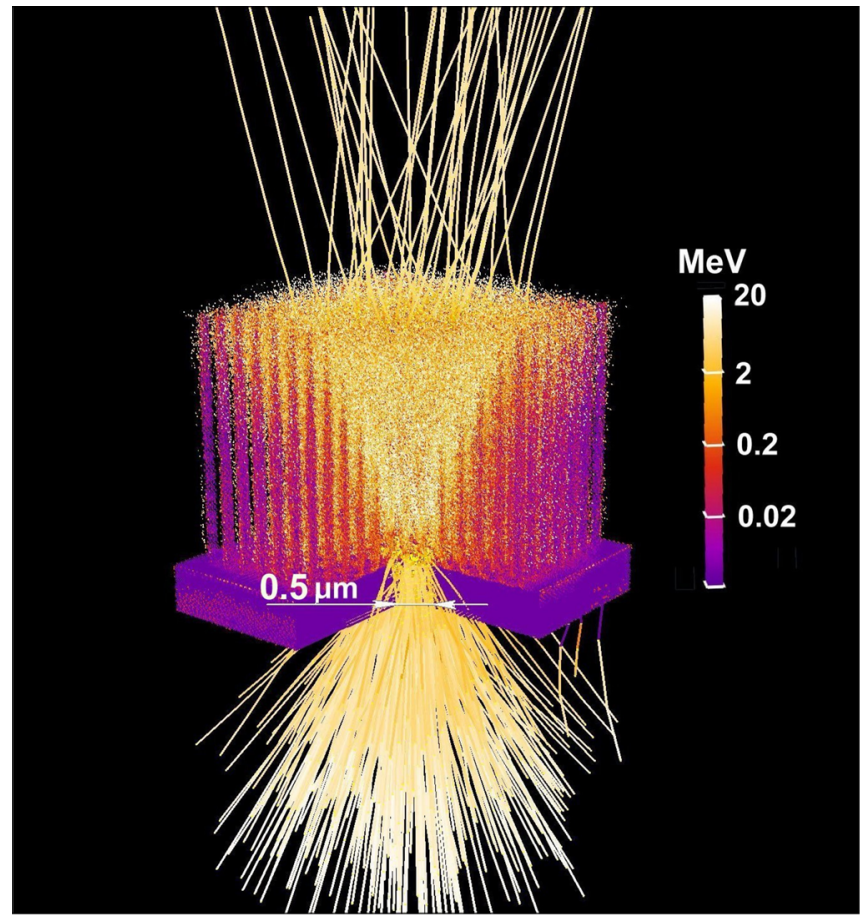

FIG. 11. PIC simulations illustrating that the use of a thin nanowire array substrate $(1 \mu \mathrm{m})$ would also result in a beam of high energy ions propagating in the forward direction. Relativistic transparency within the nanowire array leads to a small forward propagating ion source size.

nificantly increases the laser field magnitude on axis after propagating just a few micrometers within the nanowire array. This increases the relativistic critical density and the gamma factor on axis by 2-3 times, causing the plasma to become even more relativistically transparent. As a result, the laser intensity reaching the substrate is amplified by almost an order of magnitude, approaching $4 \times 10^{22} \mathrm{~W} \mathrm{~cm}^{-2}$. Enhancing electron or proton acceleration by this process of self-focusing will require optimization of nanowire density and length, as well as the substrate parameters. The creation of such forward propagating beams will require a substantially thinner nanowire substrate than those in the targets used in the present experiment.

\section{CONCLUSIONS}

The irradiation of aligned nanowire arrays of $\mathrm{CD}_{2}$ nanowires with femtosecond pulses of relativistic intensities up to $3 \times 10^{21} \mathrm{~W} \mathrm{~cm}^{-2}$ was measured to result in well collimated deuteron/proton beams with energies up to $13 \mathrm{MeV}$ propagating in the laser backward direction. At these highly relativistic intensities the tip of the nanowires rapidly explodes and the interwire gaps fill with an overdense plasma prior to the arrival of the peak of the laser pulses. However, relativistic transparency allows the laser pulses to penetrate the array all the way to the substrate. PIC simulations show that the ion beam propagating in the laser backward direction originates from ions from the exploding nanowire tips that gain energy in a TNSA sheet electric field that develops on the front of the nanowire array. Further away from the nanowire tips, in 
the central part of the nanowires, a radial TNSA field that surrounds each of the nanowires accelerates ions radially to high energy. The resulting deuteron-deuteron collisions and those of deuterons colliding with the $\mathrm{CD}_{2}$ substrate are measured to produce bursts of up to $1.2 \times 10^{7} 2.45 \mathrm{MeV}$ D-D fusion neutrons per shot. The well collimated ion beams measured here could be used in a pitcher-catcher configuration with a conversion target such as Be to produce directed beams of higher energy neutrons. Simulations also predict that the use of a thinner substrate would result in the generation of an ion beam propagating in the forward direction emanating from a small area and accelerated in a back surface TNSA field.

\section{ACKNOWLEDGMENTS}

This work was supported by the Air Force Office of Scientific Research under Award No. FA9550-17-1-0278 and by DOD Vannevar Bush Faculty Fellowship ONR award N000142012842. The experiments made use of CSU's ALEPH laser facility supported by LaserNet US DESC0021246 from the U.S. Department of Energy, Office of Science, Fusion Energy Sciences. The simulations were conducted using the CU-CSU Summit High Performance Computer System, NSF Grant No. ACI-1532235.
[1] A. P. Fews, P. A. Norreys, F. N. Beg, A. R. Bell, A. E. Dangor, C. N. Danson, P. Lee, and S. J. Rose, Plasma Ion Emission from High Intensity Picosecond Laser Pulse Interactions with Solid Targets, Phys. Rev. Lett. 73, 1801 (1994).

[2] B. Luther-Davies and J. L. Hughes, Observations of MeV ions emitted from a laser produced plasma, Opt. Commun. 18, 351 (1976).

[3] R. A. Snavely, M. H. Key, S. P. Hatchett, T. E. Cowan, M. Roth, T. W. Phillips, M. A. Stoyer, E. A. Henry, T. C. Sangster, M. S. Singh, S. C. Wilks, A. MacKinnon, A. Offenberger, D. M. Pennington, K. Yasuike, A. B. Langdon, B. F. Lasinski, J. Johnson, M. D. Perry, and E. M. Campbell, Intense High-Energy Proton Beams from Petawatt-Laser Irradiation of Solids, Phys. Rev. Lett. 85, 2945 (2000).

[4] E. L. Clark, K. Krushelnick, J. R. Davies, M. Zepf, M. Tatarakis, F. N. Beg, A. Machacek, P. A. Norreys, M. I. K. Santala, I. Watts, and A. E. Dangor, Measurements of Energetic Proton Transport through Magnetized Plasma from Intense Laser Interactions with Solids, Phys. Rev. Lett. 84, 670 (2000).

[5] A. Maksimchuk, S. Gu, K. Flippo, D. Umstadter, and V. Y. Bychenkov, Forward Ion Acceleration in Thin Films Driven by a High-Intensity Laser, Phys. Rev. Lett. 84, 4108 (2000).

[6] S. C. Wilks, A. B. Langdon, T. E. Cowan, M. Roth, M. Singh, S. Hatchett, M. H. Key, D. Pennington, A. MacKinnon, and R. A. Snavely, Energetic proton generation in ultra-intense lasersolid interactions, Phys. Plasmas 8, 542 (2001).

[7] S. J. Gitomer, R. D. Jones, F. Begay, A. W. Ehler, J. F. Kephart, and R. Kristal, Fast ions and hot electrons in the laser-plasma interaction, Phys. Fluids 29, 2679 (1986).

[8] S. C. Wilks and W. L. Kruer, Absorption of ultrashort, ultraintense laser light by solids and overdense plasmas, IEEE J. Quantum Electron. 33, 1954 (1997).

[9] F. Wagner, O. Deppert, C. Brabetz, P. Fiala, A. Kleinschmidt, P. Poth, V. A. Schanz, A. Tebartz, B. Zielbauer, M. Roth, T. Stöhlker, and V. Bagnoud, Maximum Proton Energy above $85 \mathrm{MeV}$ from the Relativistic Interaction of Laser Pulses with Micrometer Thick $\mathrm{CH}_{2}$ Targets, Phys. Rev. Lett. 116, 205002 (2016).

[10] A. Higginson, R. J. Gray, M. King, R. J. Dance, S. D. R. Williamson, N. M. H. Butler, R. Wilson, R. Capdessus, C. Armstrong, J. S. Green, S. J. Hawkes, P. Martin, W. Q. Wei, S. R. Mirfayzi, X. H. Yuan, S. Kar, M. Borghesi, R. J. Clarke, D. Neely, and P. McKenna, Near-100 MeV protons via a laser- driven transparency-enhanced hybrid acceleration scheme, Nat. Commun. 9, 724 (2018).

[11] A. P. L. Robinson, M. Zepf, S. Kar, R. G. Evans, and C. Bellei, Radiation pressure acceleration of thin foils with circularly polarized laser pulses, New J. Phys. 10, 13021 (2008).

[12] B. Qiao, M. Zepf, M. Borghesi, B. Dromey, M. Geissler, A. Karmakar, and P. Gibbon, Radiation-Pressure Acceleration of Ion Beams from Nanofoil Targets: The Leaky Light-Sail Regime, Phys. Rev. Lett. 105, 155002 (2010).

[13] T. Esirkepov, M. Borghesi, S. V. Bulanov, G. Mourou, and T. Tajima, Highly Efficient Relativistic-Ion Generation in the Laser-Piston Regime, Phys. Rev. Lett. 92, 175003 (2004).

[14] A. P. L. Robinson, R. M. G. M. Trines, N. P. Dover, and Z. Najmudin, Hole-boring radiation pressure acceleration as a basis for producing high-energy proton bunches, Plasma Phys. Controlled Fusion 54, 115001 (2012).

[15] V. A. Vshivkov, N. M. Naumova, F. Pegoraro, and S. V. Bulanov, Nonlinear electrodynamics of the interaction of ultraintense laser pulses with a thin foil, Phys. Plasmas 5, 2727 (1998).

[16] S. Palaniyappan, B. M. Hegelich, H. C. Wu, D. Jung, D. C. Gautier, L. Yin, B. J. Albright, R. P. Johnson, T. Shimada, S. Letzring, D. T. Offermann, J. Ren, C. Huang, R. Hörlein, B. Dromey, J. C. Fernandez, and R. C. Shah, Dynamics of relativistic transparency and optical shuttering in expanding overdense plasmas, Nat. Phys. 8, 763 (2012).

[17] J. C. Fernández, D. Cort Gautier, C. Huang, S. Palaniyappan, B. J. Albright, W. Bang, G. Dyer, A. Favalli, J. F. Hunter, J. Mendez, M. Roth, M. Swinhoe, P. A. Bradley, O. Deppert, M. Espy, K. Falk, N. Guler, C. Hamilton, B. M. Hegelich, D. Henzlova et al., Laser-plasmas in the relativistic-transparency regime: Science and applications, Phys. Plasmas 24, 56702 (2017).

[18] B. J. Albright, L. Yin, K. J. Bowers, B. M. Hegelich, K. A. Flippo, T. J. T. Kwan, and J. C. Fernández, Relativistic Buneman instability in the laser breakout afterburner, Phys. Plasmas 14, 94502 (2007).

[19] L. Yin, B. J. Albright, K. J. Bowers, D. Jung, J. C. Fernández, and B. M. Hegelich, Three-Dimensional Dynamics of Breakout Afterburner Ion Acceleration Using High-Contrast Short-Pulse Laser and Nanoscale Targets, Phys. Rev. Lett. 107, 045003 (2011).

[20] T. Ditmire, J. Zweiback, V. P. Yanovsky, T. E. Cowan, G. Hays, and K. B. Wharton, Nuclear fusion from explosions of 
femtosecond laser-heated deuterium clusters, Nature (London) 398, 489 (1999).

[21] S. Karsch, S. Düsterer, H. Schwoerer, F. Ewald, D. Habs, M. Hegelich, G. Pretzler, A. Pukhov, K. Witte, and R. Sauerbrey, High-Intensity Laser Induced Ion Acceleration from HeavyWater Droplets, Phys. Rev. Lett. 91, 015001 (2003).

[22] C. Zulick, F. Dollar, V. Chvykov, J. Davis, G. Kalinchenko, A. Maksimchuk, G. M. Petrov, A. Raymond, A. G. R. Thomas, L. Willingale, V. Yanovsky, and K. Krushelnick, Energetic neutron beams generated from femtosecond laser plasma interactions, Appl. Phys. Lett. 102, 124101 (2013).

[23] M. Kaluza, J. Schreiber, M. I. K. Santala, G. D. Tsakiris, K. Eidmann, J. Meyer-ter-Vehn, and K. J. Witte, Influence of the Laser Prepulse on Proton Acceleration in Thin-Foil Experiments, Phys. Rev. Lett. 93, 045003 (2004).

[24] F. Mackenroth, A. Gonoskov, and M. Marklund, ChirpedStanding-Wave Acceleration of Ions with Intense Lasers, Phys. Rev. Lett. 117, 104801 (2016).

[25] D. Margarone, O. Klimo, I. J. Kim, J. Prokůpek, J. Limpouch, T. M. Jeong, T. Mocek, J. Pšikal, H. T. Kim, J. Proška, K. H. Nam, L. Štolcová, I. W. Choi, S. K. Lee, J. H. Sung, T. J. $\mathrm{Yu}$, and G. Korn, Laser-Driven Proton Acceleration Enhancement by Nanostructured Foils, Phys. Rev. Lett. 109, 234801 (2012).

[26] T. Ceccotti, A. Lévy, H. Popescu, F. Réau, P. D’Oliveira, P. Monot, J. P. Geindre, E. Lefebvre, and P. Martin, Proton Acceleration with High-Intensity Ultrahigh-Contrast Laser Pulses, Phys. Rev. Lett. 99, 185002 (2007).

[27] V. Y. Bychenkov, P. K. Singh, H. Ahmed, K. F. Kakolee, C. Scullion, T. W. Jeong, P. Hadjisolomou, A. Alejo, S. Kar, M. Borghesi, and S. Ter-Avetisyan, Ion acceleration in electrostatic field of charged cavity created by ultra-short laser pulses of 1020-1021 W/cm², Phys. Plasmas 24, 10704 (2017).

[28] M. Roth, D. Jung, K. Falk, N. Guler, O. Deppert, M. Devlin, A. Favalli, J. Fernandez, D. Gautier, M. Geissel, R. Haight, C. E. Hamilton, B. M. Hegelich, R. P. Johnson, F. Merrill, G. Schaumann, K. Schoenberg, M. Schollmeier, T. Shimada, T. Taddeucci et al., Bright Laser-Driven Neutron Source Based on the Relativistic Transparency of Solids, Phys. Rev. Lett. 110, 044802 (2013).

[29] A. Curtis, C. Calvi, J. Tinsley, R. Hollinger, V. Kaymak, A. Pukhov, S. Wang, A. Rockwood, Y. Wang, V. N. Shlyaptsev, and J. J. Rocca, Micro-scale fusion in dense relativistic nanowire array plasmas, Nat. Commun. 9, 1077 (2018).

[30] M. A. Purvis, V. N. Shlyaptsev, R. Hollinger, C. Bargsten, A. Pukhov, A. Prieto, Y. Wang, B. M. Luther, L. Yin, S. Wang, and J. J. Rocca, Relativistic plasma nanophotonics for ultrahigh energy density physics, Nat. Photonics 7, 796 (2013).

[31] R. Hollinger, S. Wang, Y. Wang, A. Moreau, M. G. Capeluto, H. Song, A. Rockwood, E. Bayarsaikhan, V. Kaymak, A. Pukhov, V. N. Shlyaptsev, and J. J. Rocca, Extreme ionization of heavy atoms in solid-density plasmas by relativistic second-harmonic laser pulses, Nat. Photonics 14, 607 (2020).

[32] C. Bargsten, R. Hollinger, M. G. Capeluto, V. Kaymak, A. Pukhov, S. Wang, A. Rockwood, Y. Wang, D. Keiss, R. Tommasini, R. London, J. Park, M. Busquet, M. Klapisch, V. N. Shlyaptsev, and J. J. Rocca, Energy penetration into arrays of aligned nanowires irradiated with relativistic intensities: Scaling to terabar pressures, Sci. Adv. 3, 3 (2017).

[33] J. Park, R. Tommasini, R. Shepherd, R. A. London, C. Bargsten, R. Hollinger, M. G. Capeluto, V. N. Shlyaptsev, M. P. Hill, V. Kaymak, C. Baumann, A. Pukhov, D. Cloyne, R. Costa, J. Hunter, S. Maricle, J. Moody, and J. J. Rocca, Absolute laser energy absorption measurement of relativistic 0.7 ps laser pulses in nanowire arrays, Phys. Plasmas 28, 023302 (2021).

[34] P. P. Rajeev, P. Taneja, P. Ayyub, A. S. Sandhu, and G. R. Kumar, Metal Nanoplasmas as Bright Sources of Hard X-Ray Pulses, Phys. Rev. Lett. 90, 115002 (2003).

[35] G. Kulcsar, D. AlMawlawi, F. Budnik, P. Herman, M. Moskovits, L. Zhao, and R. Marjoribanks, Intense Picosecond X-Ray Pulses from Laser Plasmas by Use of Nanostructured "Velvet" Targets, Phys. Rev. Lett. 84, 5149 (2000).

[36] R. Hollinger, C. Bargsten, V. N. Shlyaptsev, V. Kaymak, A. Pukhov, M. G. Capeluto, S. Wang, A. Rockwood, Y. Wang, A. Townsend, A. Prieto, P. Stockton, A. Curtis, and J. J. Rocca, Efficient picosecond $\mathrm{x}$-ray pulse generation from plasmas in the radiation dominated regime, Optica 4, 1344 (2017).

[37] S. Jiang, L. L. Ji, H. Audesirk, K. M. George, J. Snyder, A. Krygier, P. Poole, C. Willis, R. Daskalova, E. Chowdhury, N. S. Lewis, D. W. Schumacher, A. Pukhov, R. R. Freeman, and K. U. Akli, Microengineering Laser Plasma Interactions at Relativistic Intensities, Phys. Rev. Lett. 116, 085002 (2016).

[38] A. Moreau, R. Hollinger, C. Calvi, S. Wang, Y. Wang, M. G. Capeluto, A. Rockwood, A. Curtis, S. Kasdorf, V. N. Shlyaptsev, V. Kaymak, A. Pukhov, and J. J. Rocca, Enhanced electron acceleration in aligned nanowire arrays irradiated at highly relativistic intensities, Plasma Phys. Controlled Fusion 62, 14013 (2019).

[39] Y. Wang, S. Wang, A. Rockwood, B. M. B. M. Luther, R. Hollinger, A. Curtis, C. Calvi, C. S. Menoni, and J. J. Rocca, $0.85 \mathrm{PW}$ laser operation at $3.3 \mathrm{~Hz}$ and high-contrast ultrahighintensity $\lambda=400 \mathrm{~nm}$ second-harmonic beamline, Opt. Lett. $\mathbf{4 2}$, 3828 (2017).

[40] M. G. Capeluto, A. Curtis, C. Calvi, R. Hollinger, V. N. Shlyaptsev, and J. J. Rocca, Deuterated polyethylene nanowire arrays for high-energy density physics, High Power Laser Sci. Eng. 9, e34 (2021).

[41] D. A. Dahl, SIMION for the personal computer in reflection, Int. J. Mass Spectrom. 200, 3 (2000).

[42] A. Pukhov, Three-dimensional electromagnetic relativistic particle-in-cell code VLPL (Virtual Laser Plasma Lab), J. Plasma Phys. 61, 425 (1999). 